\title{
Den (religiösen) Film? Den gibt es noch gar nicht!
}

\author{
von Johan G. Hahn
}

Die Nacht war feuchtwarm. Unruhig drehte sich der Filmhändler L.K. in seinem Bett. Absurde Gedanken behinderten den gesunden Schlaf, den er so nötig brauchte. Die gestrige Lagebesprechung mit der Führung seines Filmimperiums war schwierig, aber fruchtbar gewesen. Einige schwache Firmenteile waren geschlossen, die Belegschaft entlassen worden, die Geschäftssituation insgesamt aber war gestärkt worden. Noch nie wurden so viele Filme seiner Firmen in deutschen Kinos gezeigt. L.K. fühlte sich als ein mächtiger Mann. Er entschied über die Gedanken vieler Millionen Bürger. Letztendlich entschied er auch darüber, ob das Volk Schund oder Qualität zu sehen bekommt. Er hätte ein glücklicher Mensch sein können, wenn der Schlaf nur käme.

Aber da war dieser eine Gedanke, der inn nicht losließ. Er hatte keine Ahnung, von woher die Stimme gekommen war; aber er hatte sie eindeutig gehört. Diese gewaltige Stimme kam von irgendwo in seinem wunderschönen Büro, obwohl er alleine war. Seine Sekretärinnen waren längst gegangen. Er sah sich gerade ein Videoband mit der Rohmontage eines neuen Kinohits an, als das Bild plötzlich vor seinen Augen verschwand und da nur noch die Stimme im Raum war:

$$
\text { Leo, Leo!' }
$$

Er schaltete den Videorecorder ab und schaute sich im Raum um. Nichts war zu sehen. Ein wenig irritiert über diese Störung, setzte er sich wieder in seinen bequemen Ledersessel und drückte die Vorwärtstaste. Kaum war der Film wieder angelaufen, da verschwand das Bild wieder und erneut war diese Stimme da. Sie schien von irgendwo hinter ihm zu kommen:

,Leo, Leo!'

Wieder stand er auf und durchsuchte nicht nur sein ganzes Büro, sondern auch das der Vorzimmerdame und den Flur; überall erwartete er den Witzbold zu finden, der seinen Namen gerufen hatte, aber er fand nichts. Verärgert ging er in sein Büro zurück. Ihm war die Lust vergangen, sich den Film voller Gewalt und Softsex weiter anzusehen. Er schaltete den Fernseher und den Videorecorder aus und schloß seinen Schreibtisch ab, als ihn die Stimme wieder bei seinem Namen rief:

\section{LLeo, Leo!'}

,Was soll das? Wer ist den da? Sag schon, was los ist! Hör auf mit den blöden Gehabe!', reagierte er jetzt empört mit erhobener Stimme.

Ich kann mich dir nicht zeigen', antwortete ihm die Stimme, aber ich gebe dir einen großen Auftrag. Du wirst meine Geschichten für die Welt sichtbar machen. Dies ist mein Auftrag an dich: Drehe Filme über mich und

Dr. Johan G. Hahn (1951) lehrte bis vor kurzem Kommunikationswissenschaften an der Katholischen Universität in Utrecht; z.Zt. arbeitet er als Kommunikationsberater und Interim-Kommunikationsmanager. 
die Geschichte meines Volkes, so wie sie im Buch der Bücher beschrieben ist. Nimm dein Geld und drehe den endgültigen Film über mich, damit alle Welt mich sehen und hören kann'.

Entsetzt über eine so große Frechheit setzte L.K. sich an seinen Schreibtisch. Er solle Bibelfilme drehen? Ausgerechnet er? Und wer war das überhaupt, der glaubte, ihm - dem großen L.K. - befehlen zu können? Wer würde das alles finanzieren? Lächerlich das ganze. Aber trotzdem wurde ihm mulmig. Er machte eine kurze Notiz und legte diese einer seiner Sekretärinnen auf den Tisch. Dann schloß er seinen Samsonitekoffer, drehte das Licht in seinem Luxusbüro aus und verließ das Bürohochhaus. Unten sah sich der Nachtportier eine neue Folge der Serie Reich und Bescheuert an. L.K. grinste: Seine Serie, sein Geld. So hatte er es gerne. Die ganze Welt sollte sich seine Filme angucken, damit er mehr und mehr verdiene. Aber gleichzeitig kroch ein unbehagliches Gefühl von hinten über seinen Rücken bis in die Haarwurzeln. Es war, als ob ihn jemand Unsichtbares beobachtete; aber es war niemand da. Nur die Stimme hallte nach in seinem Kopf. Dieser Auftrag ...! Er lief in die Tiefgarage, startete sein Auto und fuhr nach Hause.

Und jetzt lag er im Bett neben seiner ruhig schlafenden Frau und konnte keinen Schlaf finden. ... Diese Stimme ...!

In diesen Monaten werden im niederländischen Fernsehen die ersten Teile der ursprünglich vom Leo Kirch-Konzern schon vor vielen Jahren angezettelten internationalen Megaproduktion zum Alten Testament gezeigt. Abraham (Joseph Sargent, I 1993) zeigt die Geschichte des Erzvaters in einem dreistündigen Film, mit Richard Harris (King Arthur in Camelot) als Abraham. Wer die biblische Geschichte über Abraham kennt, dem wird klar sein, daß sehr viel wilde Phantasie den Bibeltext hat ,aufbessern' müssen, um dem Film seine Substanz geben zu können. Das Resultat ist ein etwas träger Film, in dem es hauptsächlich um die zwischenmenschlichen Verhältnisse der Protagonisten geht: Abraham und Terach, Abraham und Sarah, Abraham und Lot, Abraham und seine Feinde usw. Das Verhältnis zwischen Gott und Abraham, um das es in den biblischen Erzählungen im wesentlichen geht, wird filmisch kaum glaubwürdig dargestellt; ebensowenig wie die ,Wunder', die im Film nicht über das alte Niveau filmischer Tricks am Schneidetisch hinauskommen. Auch Gott selber ist im Film entweder mittels der klassischen Stimme im ,off oder aber mittels eines Mannes, dessen Gesicht von einer Kapuze verdeckt wird, wenig überzeugend dargestellt. Beide Darstellungsweisen scheinen eher aus einer Verlegenheit heraus geboren: In der Filmgeschichte ist Gott kaum jemals glaubwürdig dargestellt worden, und auch diese Serie enttäuscht in diesem Punkt.

Die theologisch so zentrale Frage nach dem ,Warum' der Reise Abrahams von Ur nach Haran und von dort nach Kanaan: die Frage nach dem ,Warum' des Vertrauens Abrahams auf diesen ,neuen' Gott, 
der das Unmögliche von ihm fordert, diese Frage wird nirgendwo im Film glaubwürdig beantwortet. Dort, wo die biblischen Geschichten den Leser zum Wunder des Glaubens hinführen, wirkt der bzw. dieser Bibelfilm wenig glaubwürdig. Es ist, als ob die Filmemacher krampfhaft versucht haben, das ,Unglaubliche' glaubhaft darzustellen. Da, wo sie scheitern, bleibt der Film eine leere Hülle.

Der kurze Aufenthalt Abrahams in Ägypten wird auf fast mosaische Weise abgebrochen: Eine Krankheitsepidemie überfällt den Pharao und zwingt ihn, seine Geliebte Sarah - der Pharao hat sie Abraham geraubt - mitsamt ihrem Volk gehen zu lassen: let my people go. Und warum die Geschichte von Abraham, die ja hier filmisch rekonstruiert sein will, erst in Haran anfängt und abrupt mit dem Opfer Isaaks endet, wird auch nicht deutlich. Allem Anschein nach fehlte es den Machern an Kreativität und den Mitteln, um den Film auf das ganze Leben Abrahams zu erweitern, denn nicht nur der Anfang der Geschichte fehlt: In der zweiten Hälfte des Filmes spricht Gott darüber, daß er Abraham aus Ur in Chaldea gerufen hat. Am Anfang des Filmes erfährt Abraham den neuen Gott aber erst in Haran, und auch das Lebensende der Erzvaters bleibt dem Zuschauer verborgen. Genauso verhält es sich mit der zweiten kürzeren Folge der Serie - Jacob'. Auch diese Folge leidet unter den typischen Problemen des Phänomens Bibelfilm: die Unfähigkeit, eine Geschichte glaubwürdig zu rekonstruieren (Landschaft, Zelte, Kostüme usw.), ohne die grundsätzliche Glaubwürdigkeit der Geschichte (Was erzählt uns die biblische Geschichte über die Zeit, in der wir jetzt leben?) zu vergewaltigen. Und genau dies tun die meisten Bibelfilme: Sie reduzieren die biblische Geschichte auf eine damalige und historisch rekonstruierbare Geschichte ohne jegliche Verbindung mit dem aktuellen Leben der Zuschauer. Alles in allem macht auch diese Serie deutlich, daß dieses Problem, genau wie in den vielen vorhergegangenen Bibelverfilmungen, noch nicht gelöst worden ist - wenn es sich denn überhaupt lösen läßt. Vielleicht können wir sagen, daß es lediglich Krzysztof Kieslowski - wie auch einigen anderen Regisseuren - mit seiner Serie Dekalog gelungen ist, die Verbindung zwischen der alten biblischen Erzählung und dem Leben des Zuschauers im Hier und Jetzt darzustellen. Dem klassischen Bibelfilm gelingt das sicherlich nicht. Und genau dieses Defizit ist ein herausragendes Merkmal nicht nur vieler Bibelfilme, sondern auch vieler Filme, die umgangssprachlich, religiöse Filme‘ genannt werden.

In allem scheint die Bibel-Serie den vergleichbaren Produktionen (z.B. Jesus won Nazareth [Franco Zeffirelli, I/GB 1976], Jesus [John Heyman, USA 1979], Die größte Geschichte aller Zeiten [George Stevens, USA 1963], König der Könige [Nicolas Ray, USA 1960] u.v.m.) sehr ähnlich zu sein. Zwar sind die technischen Möglichkeiten im Vergleich zu früheren Bibelfilmen weitaus besser geworden, aber auf dem Gebiet der Dramaturgie, der Inszenierung und der Kostümierung haben die Bibelfilmer anscheinend wenig dazugelernt. Noch immer scheint es so zu sein, daß ein Bibelfilm eine Art Kreuzung zwischen einem 
bewegenden Gemälde von Rembrandt, einer Comicserie, einem NahOst-Kostümfilm der 50er Jahre und den frommen methodistischen Jesusbildern, wie z.B. Thomas Hardy sie im 19. Jahrhundert malte, sein muß. Obwohl jüdische, christliche und islamische Theologen dem Projekt als Berater zur Seite standen, haben rein äußerlich weder die neuesten Ergebnisse der alttestamentlichen Forschung noch der Archälogie oder sonstige neue Erkenntnisse der Bibelforschung einen sichtbaren Einfluß auf das Drehbuch gehabt. Und wenn dieses Wissen dennoch irgendwie in das Drehbuch eingeflossen ist, so wurde dies allem Anschein nach überlagert von den bekannten alten ikonologischen Vorurteilen und Klischees, die den meisten Bibelfilmen anhaften. Die filmischen Bibelgestalten zeigen kaum eine Evolution, als ob wir heute, wie schon vor 100 Jahren wußten, wie sie ausgesehen haben.

Damit will ich nicht behaupten, daß die Filme ungenau oder gar schlecht gemacht sind. Im Gegenteil: Die drei Folgen der Serie, die ich bis jetzt gesehen habe, sind fachmännisch einwandfrei hergestellte Produktionen, für die sich Hollywood sogar im B-Film-Bereich nicht zu schämen braucht. Und auch die enormen Lücken in den ursprünglichen Bibelgeschichten sind mit viel Geschick, also filmisch glaubhaft, von den Drehbuchautoren geschlossen worden, aber dennoch irgendwie zu unwirklich, um realistisch und daher glaubwürdig sein zu können. Von Abraham und Jacob wissen wir aus den biblischen Erzählungen zwar relativ viel, aber es bleibt auf jeden Fall zu wenig für eine eingehende Rekonstruktion ihres Lebens. Und das ist genau das, was die Macher dieser Filmart immer wieder versuchen. Sie hätten damals gerne eine Filmkamera zugegen gehabt, aber da dies nicht möglich war, rekonstruieren sie die damalige Zeit eher schlecht als recht und glauben so, die biblische Geschichte zu ihrem Recht kommen lassen zu können. Da entwirft die allzu menschliche Phantasie der Filmemacher schon mal einiges, was es in Wirklichkeit wahrscheinlich so gar nicht gegeben hat. $\mathrm{Da}$ werden die großen Geister der Erzväter zu fast kleinbürgerlichen Menschen. Da wird Geschichte mit allen nur denkbaren Mitteln ,rekonstruiert' und der Zuschauer bekommt den festen Eindruck, daß im Film alles genauso aussieht, wie es damals gewesen ist. Dem Zuschauer wird die filmische Phantasie der Filmemacher als eine Art von Semi-Realismus vorgehalten; aber er kann die Grenze zwischen Phantasie und Wirklichkeit nur sehr schwer wahrnehmen. Nirgendwo enthalten die Filme einen Hinweis auf das Verhältnis zwischen der Wirklichkeit der biblischen Erzählung und der Phantasie der Filmemacher. Somit wird dem Zuschauer eine ganz andere Geschichte erzählt, als er sie in der Bibel lesen kann: eine neue Geschichte, die genauso von der ursprünglichen Geschichte zu unterscheiden, so anders und so selbständig ist, wie z.B. Boris Pasternaks Roman Doktor Schiwago von der Filmfassung David Leans (USA 1965) zu diesem Roman. Beide Produkte sind eigenständige Kunstwerke mit nur peripheren Verbindungen. So ist es meistens mit Literaturverfilmungen: entweder das Buch ist gut und der Film schlecht oder umgekehrt. Nur selten halten sich beide im Gleichgewicht. Und noch seltener ähneln sich Literatur- 
vorlage und Film so sehr, daß von einer echt gelungenen Unsetzung des einen Mediums in das andere gesprochen werden kann. Meistens leidet das Buch unter seiner Verfilmung. Und dann stellt sich ganz grundsätzlich die Frage nach dem Verhältnis zwischen den biblischen Geschichten und den vielen Bibelfilmen.

Die Frage bleibt also, wo bei dieser Bibelserie der entscheidende Unterschied zwischen einer Bibelverfilmung und der Verfilmung eines willkürlichen anderen Buches der Weltliteratur liegt. Hier wird, laut Pressemitteilung, die ,endgültige‘ Verfilmung der Bibel vorgestellt. Das Wissen über dieses viel umkämpfte Buch und 100 Jahre Filmgeschichte soll im Zusammenspiel mit dem Können der Filmfabriken eine Art audiovisueller Superbibel ergeben, um sie der Welt anzubieten. Da können die Erwartungen hoch angesetzt, aber die Entäuschung umso größer sein. Ich neige eher zu letzterem: Selbstverherrlichende Superlative haben auf mich noch nie einen großen Eindruck gemacht. Erst recht nicht, wenn es um Bibelfilme geht. Der Grund dafür ist, daß, wer die Geschichten der Bibel nicht kennt, wahrscheinlich auch in die Filme nichts ,Glaubhaftes' hineinlegen kann. Die grundsätzliche Verbindung zwischen der filmischen Geschichte und der zugrundeliegenden biblischen Geschichte kann nicht gelingen. Dem nicht christlich-religiös erzogenen Zuschauer bleiben im besten Fall Erinnerungen an irgendwelche merkwürdigen Geschichten kostümierter Menschen. Ein grundsätzliches Kennenlernen der heilvollen Bemühungen Gottes mit seinem auserwählten Volk wird über derartige Filme kaum zustande kommen können. Dazu ist weit mehr nötig als nur ein Film. Notwendig wäre nämlich der direkte menschliche Kontakt, der die alten Geschichten aktualisiert und fühlbar werden läßt, was, glauben' heißt. Gerade diese Aktualisierung der Geschichten ist es, die in dieser Art von Filmen fehlt. Der zeitgenössische Zuschauer wird sich zurecht fragen: ,Was gehen mich diese alten Geschichten an? Was haben die mit meiner jetzigen Lage zu tun?' Der Bibelfilm, auch diese neueste Serie, kann - aus seinem ,rekonstruktionistischen Konzept' heraus - darauf keine Antwort geben. Nicht das bloße Erzählen der Geschichte ist vorrangig, sondern das Aktualisieren. Das Neubeleben der alten Erzählungen sollte im Vordergrund stehen. Wer nur diese Filme sieht, läuft Gefahr, sich in seiner Phantasie vor allem an die Filmbilder zu erinnern, die eine weitaus tiefere Wirkung als die Bilder einer früheren Kinderbibel haben. Die filmischen Bilder sind nämlich auf Dauer nicht mehr von anderen filmischen Wirklichkeitsbildern zu unterscheiden. Sie erhalten einen hohen ,Wirklichkeitswert'; und es ist die Frage, ob das die Absicht dieser Produktion ist. In einer multi-religiösen und multi-konfessionellen Gesellschaft haben die vielen inneren religiösen Bilder ein eigenes Existenzrecht. Wer mit Hilfe einer Bibelfilmserie anno 1995 (und später) diese Bilderfreiheit einzudämmen versucht, lädt das Risiko auf sich, ,religiös bildlich' mit Hilfe völlig ,unechter' Bibelbilder zu indoktrinieren. Und das kann nicht der Sinn einer solchen Serie sein! Die Macher müssen davon ausgehen, daß ein Großteil der Zuschauer - zumindest in der westlichen Welt - die biblischen Geschichten nicht mehr kennen. 
Die fortschreitende Dekonfessionalisierung (Stichwort ,Kirchenaustritte') der aufgeklärten westlichen Gesellschaft macht die biblischen Geschichten zu ,Minderheitengeschichten'. Und anderswo auf der Welt gibt es zu den biblischen Geschichten kulturspezifische Bilder, die denen der Bibelfilme nicht gerecht werden.

Wer die biblischen Geschichten noch kennt, kann auf die neue Serie im Prinzip in zweierlei Weise reagieren: totale Zuneigung, eine Reaktion, die der Niederländische Christliche Fernsehsender (NCRV), der die internationale Co-Produktion mitproduziert, gerne schüren würde; oder aber Ablehnung, weil die eigenen Bilder zu den Geschichten anders sind, verwurzelt in der eigenen Kultur, ursprünglicher, menschlicher, weniger künstlich wie die im Film und auch weil die Filmbilder nichts mit dem eigenen Leben am Ende des 20. Jahrhunderts zu tun haben. Es sind fremde leere Bilder, ohne jegliche Verbindung mit dem eigenen Leben. $\mathrm{Ob}$ es bei denen, die die Geschichten nicht kennen - und in den Niederlanden gibt es eine schnell wachsende Zahl Jugendlicher, die ohne die Bibelgeschichten erzogen worden sind, was im schulischen Religionsunterricht oft $\mathrm{zu}$ Verständnisschwierigkeiten führt -, eine Zuneigung geben wird, bleibt noch eine offene, aber wohl eher negativ $\mathrm{zu}$ beantwortende Frage. Dies ist für mich jedoch die zentrale Frage, wenn es darum geht, ob Bibelfilme sinnvoll sind. Jeder Produzent weiß, $\mathrm{da} ß$ er mit diesem Genre nicht viel verdienen wird; also muß es andere Motive geben, warum eine internationale Produktionsgemeinschaft unter anderem mit Geldern von Ted Turner (CNN) mehr als 120 Mio. Dollar in 21 Filme zum Alten Testament investiert.

Die tieferliegenden Gründe der Produzenten und Mitarbeiter kenne ich nicht - ich habe ein mögliches Motiv zu Beginn dieses Beitrages in etwas alttestamentlicher Art und Weise' paraphrasiert -, aber wenn eine derart teure und langlaufende Produktion (Produktionsplanung mehr als 5 Jahre!) einen Sinn haben will, so kann dieser nur im religiösen und nicht im wirtschaftlichen Bereich gesucht werden. Das heißt im Klartext, daß die Serie vor allem der religiösen Bildung auf einer internationalen und überkulturellen Ebene dienen soll. Die Frage ist aber, ob eine solche moderne Fassung der klassischen Bildergeschichten (Comic, Bande dessinée) zu den biblischen Geschichten, diejenige ist, die der moderne kultur-unabhängige Religionsunterricht braucht. Erreicht die Serie überhaupt die Jugend, wo auch immer auf der Welt? Für wen ist sie sonst gemacht? Für Erwachsene? Für die, die schon glauben? Als ein modernes Hilfsmittel zur Evangelisation? Funktioniert sie in den Vereinigten Staaten anders als in Europa, und anders als in Afrika oder Asien? Wird sie in den USA vielleicht von der ,Neuen Christlichen Rechten' im Kampf gegen den ,Unglauben', als Mittel im politischen Kampf benutzt? Wäre eine Multimedia-Serie mit kurzen Filmen, mit Arbeitsmaterialien, Büchern, CD-ROMs, Videocassetten, Tonträgern usw. nicht viel hilfreicher gewesen? Es gibt noch sehr viele Fragen zu der Serie, aber nur wenige Antworten. Geht man von dem Diktum ,Wer glaubt, braucht keinen Beweis; für den, der nicht glaubt, 
ist jeder Beweis sinnlos' aus, dann ist die Serie eine eher fragwürgige Investition.

\section{II.}

Diesen etwas längeren Anlauf $\mathrm{zu}$ meinem Thema brauchte ich aus aktuellem Anlaß', denn diese Bibelfilmserie zeigt, daß sich auf dem Gebiet des ,religiösen Films' einiges tut. Und dennoch bin ich der Meinung, daß es den ,religiösen Film' an sich gar nicht gibt. Wie schon erwähnt, unterschätzt der Begriff, religiöser Film' den Einfluß des Zuschauers auf die Bedeutungsdefinition eines Filmes.

Überlassen wir daher die konkrete Diskussion um diese BibelfilmSerie den christlichen Filmkritikern und wenden uns der Grundsatzfrage $\mathrm{zu}$, ob es überhaupt einen religiösen Film gibt. Um diese Frage eingehend beantworten zu können, möchte ich zuerst das Phänomen 'Film' als solches in seinen Verhältnis zu anderen vergleichbaren Medien kurz umreißen. Danach werden wir uns mit der Frage nach einigen, als religiös verstandenen Filmkategorien auseinandersetzen, um zuletzt zu zeigen, wie im Prinzip jeder Film zu einem religiösen Film werden kann.

Wenn ich ins Theater oder in die Oper gehe, dann weiß ich, was mir geboten wird. Akteure spielen Rollen und bieten mir in dem Raum, in dem ich mich befinde, ein Schauspiel, an dem ich sozusagen unmittelbar Teil habe. Ich kann Teil der dramatischen Handlung werden - in vielen zeitgenössischen Theatervorstellungen wird der Zuschauer in die Handlung einbezogen - er kann in die Handlungen eingreifen und sie verändern. Jede Vorstellung ist ein einzigartiger und unwiederholbarer Handlungskomplex. Jeder Regisseur macht aus einem Theaterstück oder einer Oper ein ganz neues Erlebnis. Sogar jede einzelne Vorstellung kann sich von den anderen Vorstellungen unterscheiden. Theater ist lebendig, Theater ist Präsenz.

Im Kino aber ist alles anders. Der Film ist tot, ist Vergangenheit. Dort gibt es nichts Lebendiges, an dem ich Teil haben könnte. Alles was ich sehe, war schon da, ist schon vorbei und wird nur noch mit Hilfe toter technischer Kunstmittel reproduziert. Zweidimensionale farbige Lichtflecke, im dunklen Raum auf eine Leinwand projiziert, wollen mir die Illusion von fernen Ländern, großen Abenteuern, abgrundtiefen Emotionen geben. Aber im Kino gibt es nichts, an dem ich Teil haben kann, außer an einem Lichtspiel, an einer Illusion. Kostspielige Mlusionen sind es, mit ihrem eigenen Reiz, Illusionen als Resultate von Milliardengeschäften. Kino ist immer Vergangenheit. Es ruft Träume im Zuschauer hervor, die es nicht realisieren, dafür aber emotional erleben lassen und kompensieren kann. Daß Film schon vor seiner Uraufführung emotional wirken kann, zeigen die Reaktionen auf einen Film wie Martin Scorsese's Letzte Versuchung Christi (USA 1988). 
Der Film an sich ist nichts. So wie das Theaterstück an sich nur aus einem von den Schauspielern reproduzierten Text besteht, so besteht der Film nur aus einem langen Kunststoffband, dem die Bilder mit Hilfe chemischer Prozesse anhaften. Der Film wird erst etwas Illusorisch-Reales, wenn Menschen sich in einen dunkeln Raum setzen und sich das Schattenspiel auf einer Leinwand ansehen. Das Kunststoffband und der Projektor machen erst das Filmerlebnis möglich. Dieses Filmerlebnis ist es, worum es im Kino geht. Die 24 Schattenbilder pro Sekunde, die der Projektor auf die Leinwand wirft und somit lebensechte Bewegung suggeriert, sind dazu da, um im Zuschauer etwas auszulösen, wodurch dieser zu einer (un)angenehmen Erfahrung kommen kann. Wer einmal Gelegenheit hatte, sich im Kino die Zuschauer und den Film gleichzeitig anzusehen, der weiß, zu welch kraftvollen und direkten Reaktionen ein Film führen kann. Die Tränen, die ein Film wie Lovestory (USA 1969) bei vielen hervorrief, waren durchaus echt und auch die typische ,Cowboy-Gangart', praktiziert von manchen Männern beim Verlassen des Kinos nach einem Western, macht klar, wie direkt ein Film manchmal ,wirkt'. Und - zum Glück nur selten - erreichen uns über die Medien Nachrichten, aus denen hervorgeht, daß irgendein schwacher Geist den Horror eines Filmes in seine eigene Welt übertragen hat. Daß viele moderne Filme genau auf das Schüren latenter Gefühle und Erfahrungen zielen, sehen wir in den Werbepostern für Filme, auf denen vielfach emotionalisierende Werbetexte erscheinen. Der Zuschauer wird über seine Emotionen ins Kino geholt.

Ein Film, so können wir feststellen, ist Handel mit meist angenehmen Erfahrungen, letztendlich vergleichbar mit der Werbebranche oder der Reisebranche. Auch dort werden Erfahrungen gehandelt. Schließlich kaufen wir keinen Kaffee, sondern genießen in gemütlicher Runde'; wir kaufen wir keine Wegwerfwindeln, sondern besorgen unserem Baby ,Spielfreiheit ohne nasses Gefühl'; und wir reisen nicht nach Indonesien, sondern erleben spannende Abenteuer im smaragdgrünen Inselreich am Äquator'. Der Film will dem Zuschauer auch eine ihm angenehme Erfahrung bieten, wobei dies durchaus eine Angstoder Horrorerfahrung sein kann, denn auch solche Erfahrungen werden von vielen Menschen als zum Teil angenehm eingestuft.

Manche Filme wollen eine ganz besondere Art von Erfahrung hervorrufen, die ich hier der Einfachheit halber eine ,religöse Erfahrung' nennen möchte. Bei einer solchen Erfahrung geht es um eine Erfahrung, von der das erfahrende Subjekt der Meinung ist, daß diese in Verbindung steht mit Elementen aus seinem religiösen Glauben, seiner Weltanschauung. Im Prinzip kann jede Erfahrung mit Elementen des religiösen Glaubens in Verbindung gebracht werden. Daran wird deutlich, daß vor allem sehr spezifische Erfahrungen religiöser Art sein oder werden können. So können z.B. bestimmte Texte oder Bilder eine Erfahrung auslösen, die wir mit etwas assoziieren, daß wir ,religiös“ nennen. Sehen wir etwa einen Mann an einem Kreuz, so assoziieren wir 
das mit einem uns bekannten, typisch christlichen Bild. Das wahrgenommene, in sich vielleicht neutrale Bild kann durch die Verbindung mit einem christlichen Bild zu einer religiösen Erfahrung führen. Wir erkennen das Bild aufgrund früherer Erfahrungen. Erfahrungen, die wir in einem bestimmten Rahmen gemacht, verarbeitet und in diesen Rahmen integriert haben. Eine voll integrierte ästhetische Erfahrung kann angesichts bestimmter Rahmenbedingungen zu so etwas wie einer religiösen Erfahrung führen. Dabei können auch rein profane, d.h. nicht religiös orientierte Bilder oder Texte als Auslöser für eine religiöse Erfahrung dienen. Wer sich in einem bestimmten vorgegebenen Rahmen einen Naturfilm im Fernsehen ansieht, kann dies durchaus zurecht als ein Bild der Schöpfung Gottes verstehen, während derselbe Film für jemand anderen ein schöner Naturfilm ohne jegliche religiöse Konnotation bleibt.

Die ,Fähigkeit", Bilder in einen Rahmen zu integrieren und dadurch zu verstehen, nenne ich die Fähigkeit zur ,erkennbaren Analogie'. Nur wer imstande ist, ein Bild zu erkennen und die Analogie zwischen diesem Bild und einem schon vorher in einem bestimmten Rahmen gesehenen und verarbeiteten Bild erkennt, kann zu dieser ,erkennbaren Analogie' kommen, und das Bild ,aufnehmen'.

Für unser Thema heißt das im Klartext: Nur wer die biblischen Geschichten schon kennt, wird dem Bibelfilm das abgewinnen können, was von den Machern beabsichtigt wird. Er erkennt die Geschichten im Film als die Geschichten, die er in einem anderen Rahmen schon mal gehört hat. Es kann dann zu einer ,religiösen Erfahrung' kommen. Für wen die erkennbare Analogie nicht zustandekommt, d.h. für den, der die biblischen Geschichten nicht kennt, ist ein Bibelfilm nur ein mehr oder wenig gelungener Film mit einer Geschichte ohne jede tiefere religiöse Bedeutung. Der von den Machern beabsichtige religiöskommunikative Prozeß scheitert an dem fehlenden Vorverständnis der Zuschauer. Und genau dort setzt meine grundsätzliche Kritik am Konzept, religiöser Film' an.

Ausgangspunkt meiner Überlegungen ist folgendes: wenn profane Bilder beim Zuschauer Assoziationen hervorrufen, die für den Zuschauer etwas mit seiner religiösen Überzeugung beziehungsweise mit seinen früheren Erfahrungen mit Religion zu tun haben, dann können diese Bilder als religiös zu interpretierende Bilder verstanden werden. Mit anderen Worten: das gleiche Bild kann bei dem einen Zuschauer zu einer religiösen oder als religiös verstandenen Erfahrung, bei dem anderen Zuschauer aber zu einer eher profanen, d.h. nicht religiös zu interpretierenden Erfahrung führen. Eine mögliche Erklärung für diesen Unterschied bietet das wahrnehmungstheoretische Konzept der ,erkennbaren Analogie'. Daraus geht hervor, daß der Zuschauer derjenige ist, der die für ihn gültige Bedeutung eines Bildes aufgrund seiner vorherigen Erfahrungen definiert. Er erkennt sozusagen eine Analogie zwischen dem aktuellen Bild und einem in seinem Gedächtnis 
gespeicherten früheren Bild und formt sich aufgrund dieser Analogie eine entsprechende Bedeutung des aktuellen Bildes. Aus der andauernden Interaktion zwischen aktuellen und gespeicherten Bildern entsteht ein komplexes Bildgedächtnis im Zuschauer, mit dem immer neue Bildbedeutungen - auch von alten Bildern - generiert werden können. Dies gilt nicht nur für die vielen Bilder, die wir tagtäglich auf uns einwirken lassen, sondern auch für jede Art von Standbildern wie Fotos, Statuen oder Gemälden, aber ebenso auch für den Film als Komposition einer Vielfalt zusammengehörender Bilder. Gehe ich also davon aus, daß es keine in sich, d.h. für jeden beliebigen Zuschauer erkennbaren religiösen Bilder gibt, sondern nur vom Zuschauer aufgrund seines Bildgedächtnisses als religiös verstandene Bilder, so ist es nur ein Schritt bis zur Feststellung, daß es auch keine religiösen Filme an sich gibt, sondern nur Filme, die religiös verstanden werden können. Damit ist aber noch nicht alles gesagt, denn es bleibt die Frage, ob es irgendwelche innerfilmischen Kriterien gibt, außerhalb derer eine solche religiöse Interpretation nicht mehr möglich ist? Anders gefragt: Gibt es ein theoretisches Feld, innerhalb dessen man sich alle Filme denken kann, die eine etwaige religiöse Interpretation erlauben? Ebenso kann man sich die Frage stellen, ob es auch ein analoges theoretisches Feld für die Filme gibt, die sich nicht religiös interpretieren lassen. Und es bleibt die Frage offen, ob sich die beiden Felder möglicherweise irgendwo berühren oder überschneiden.

\section{III.}

Um diese Fragen beantworten zu können, müssen wir tiefer in die Eigenart des Filmes vordringen. Dabei gehe ich von einer ganz bestimmten Perspektive aus. Sehen wir uns einen willkürlichen Film an, so können wir zwischen zwei grundsätzlich zu unterscheidenden ,Versionen' eines einzelnen Filmes unterscheiden. Auf der einen Seite gibt es den Film, so wie er vom Filmemacher und seinem Team hergestellt wurde. In dieser Version enthält der Film die Bilder und Dialoge, Musik und Handlungen, wie sie der Filmemacher gewollt hat. Es ist sein (bzw. ihr kollektives) Produkt, das in vielen Kopien von tausenden von Menschen weltweit gesehen werden kann. Diese Version eines Filmes nenne ich die ,Produktiv-Version'. Es ist die ursprüngliche Version des Filmes, so wie sie konzipiert und hergestellt worden ist und gibt ihrer ,produktiven' Art nach grundsätzlich die Interpretationsgrenzen für den Zuschauer an. Es ist auch die Version, die der Zuschauer in projizierter Form, sieht'.

Es gibt aber noch eine andere, multiple Version, die zwar teilweise abhängig ist von der urspünglichen Version, sich aber dennoch deutlich davon unterscheiden läßt. Es ist die Version, die der Zuschauer aufgrund seines Film-'sehens' wahrnimmt, d.h. die Version, die er aus der "Produktiv-Version' heraus für sich rekonstruiert, nicht nur auf Grund seines aktuellen Film-sehens, sondern auch aufgrund seiner 
früher gespeicherten Erfahrungs- und Erinnerungsbilder. Das oben erwähnte Konzept der ,erkennbaren Analogie' macht klar, warum ein und derselbe Film bei verschiedenen Zuschauern zu unterschiedlichen Wahrnehmungen und somit zu unterschiedlichen Erfahrungen führen wird. Wer schon einmal mit einer Gruppe im Kino war und sich nachher den Film gegenseitig nacherzählt hat, weiß über die Unterschiedlichkeit der verschiedenen Nacherzählungen zu berichten. Wir können daher sagen, daß die ,Produktiv-Version' eines Filmes zu vielen ,Rezeptiv-Versionen' führen wird. Für den einzelnen Zuschauer gibt es nur seine eigene ,Rezeptiv-Version', von der aus sich die "ProduktivVersion` nicht einfach rekonstruieren läßt.

Die ,erkennbare Analogie' macht es einem Zuschauer möglich, einen Film zu erfahren, wenn es für ihn im Film erkennbare Bilder, Geräusche und Situationen gibt. Ist das der Fall, und kann er diese Bilder adäquat ordnen und verstehen, dann erhält der Film eine für den Zuschauer kohärente Bedeutung. Dies kann sowohl eine profane wie auch eine religiöse Bedeutung sein, je nachdem, ob die analogen Bilder, die der Film hervorruft, einem profanen oder religiösen Bereich zugeordnet werden. Diese Wahl trifft der Zuschauer eher unbewußt. Ob für ihn Bilder religiös oder profan verstanden werden, hängt zum größten Teil von einer Vielzahl seiner früheren Bilder und Erfahrungen ab.

Für den ,religiösen Film' als ,Filmgenre' - ich bin allerdings der Meinung, daß es sich hier nicht um ein eigenes Genre handelt -, heißt dies, daß eine nähere Analyse dieser Art von Filmen notwendig ist, ehe wir feststellen können, zu welcher ,erkennbaren Analogie' derartige Filme führen können. Ist der ,religiöse Film' eine spezielle Art von Film, also ein eigenes Genre, so sollte es möglich sein, die typischen unterschiedlichen Merkmale dieser Art von Filmen festzulegen, so daß eine eindwandfreie und eindeutige Identifikation des Genres möglich wird. Genau da finden wir aber das größte ungelöste Problem. Die Analyse einer großen Anzahl von Filmen zeigt, daß es den produktivreligiösen Film durchaus geben kann. In einen solchen Film wird Religion bzw. Religiöses als Thematik verarbeitet und manifest. Wir sehen Gegenstände, Handlungen und Personen, die innerhalb eines bestimmten kulturellen Umfeldes als religiöse Gegenstände, religiöse Handlungen oder religiöse Personen verstanden werden können (z.B. Kirchengebäude, Kruzifix, Weihwasserbecken, Ka'aba in Mekka, Talar, Taufe, Priesterweihe, betende Muslime, Nonnen, Papst usw.). Aber, und das sollte mit Nachdruck gesagt werden, es handelt sich hier um Inhalte, die nur vom Zuschauer auf der Basis einer, erkennbaren Analogie' als religiöse Inhalte erkannt werden können. Der Zuschauer muß die Gegenstände, Handlungen und Personen schon einmal als religiös definiert haben. Fehlt diese ,erkennbare Analogie', dann wird der Zuschauer das - vom Verständnis des Filmemachers als manifest religiös Dargestellte - nicht als solches wahrnehmen können. Der Zuschauer wird den entsprechenden Film(abschnitt) nicht als religiös verstehen können. 
Es gibt auf der anderen Seite aber auch den rezeptiv-religiösen Film. Dieser kann durchaus produktiv-profan sein, also keine religiöse Thematik aufweisen, aber trotzdem eine religiöse Erfahrung beim Zuschauer abrufen.

Wir können uns diese Situation am besten an einigen Beispielen klarmachen:

- Im Film Viva Maria von Louis Malle (I/F 1965) kommt eine Szene vor, in der Maria eine vorbeiziehende Gruppe gefangener Freiheitskämpfer beobachtet, die aneinandergekettet sind. Einem der Gefangenen - der Führer der Freiheitskämpfer - hat man zur Strafe einen Balken quer auf den Rücken gebunden und seine Arme mit Tauen an den Balken befestigt. Es ist für einen Zuschauer, der auch nur wenig mit dem Christentum vertraut ist, naheliegend, in dieser Figur den ,Christus auf dem Kreuzweg' zu erkennen. Es ist daher ohne weiteres möglich, zumindest Teilen dieses Filmes eine (vom Zuschauer her gesehen) religiöse Bedeutung beizumessen, die indes vom Hersteller des Filmes vielleicht gar nicht als solche gemeint ist. Der Zuschauer sieht die Bilder, interpretiert sie im Rahmen seines eigenen Bildverständnisses und nimmt darin etwas wahr, was sozusagen aus ihm selber kommt. Der Zuschauer macht seinen eigenen ,Rezeptiv-Film', der prinzipiell anderer Art sein kann als die profane ,Produktiv-Version'.

- Ein zweites Beispiel entnehme ich dem Film Willow von Ron Howard (USA 1987). Am Anfang des Filmes wird erzählt, wie eine böse Königin alle Babys umbringen läßt, weil einer Vorhersage nach, eines davon ihre Macht brechen wird. Wenig später prophezeit die Mutter eines der Babys, daß es ihr Kind sein wird, das das Böse überwinden und die Macht der Königin vernichten wird. Mit Hilfe einer Geburtshelferin wird das Kind gerettet und am Fluß in ein Schilfkörbchen gelegt, wo es von Zwergen gefunden und gerettet wird. Am Ende des Filmes wird die böse Königin durch die Zaubermacht von Willow Ufgood, dem Zwerg, der es als seine Aufgabe versteht, das Baby zu retten, vernichtet. Es braucht für den, der die biblischen Geschichten kennt, wenig Phantasie, um hier deutliche Verweise auf die Maria- und Christusfigur und auf das Kind Moses, das auch aus einem Binsenkörbchen gerettet wird, zu sehen. Wem aber diese Bilder in der Erinnerung fehlen, wird diese Verbindung mit den biblischen Figuren nicht zustande bringen können. Für ihn bleibt der Film ein unterhaltender profaner Phantasiefilm, ohne jede religiöse Beziehung. Das Fehlen einer erkennbaren Analogie fürt zu einem anderen nicht-religiösen ,Rezeptiv-Film'.

- Ein drittes Beispiel liefert uns der Film Terminator II von James Cameron (USA 1991). In diesem Film tritt der Humanoid ,Terminator II' (Arnold Schwarzenegger) mit dem Auftrag auf, die Welt vor dem 
Untergang zu retten'. Er hat diesen Auftrag, weil er aus der Zukunft kommend in der Vergangenheit die Geschichte beinflussen soll, damit nicht das eintritt, was geschähe, wenn er die Geschichte nicht beinflussen würde. Er soll sozusagen mit seinem Wissen über die Zukunft der Erde diese Zukunft anders und damit natürlich besser gestalten helfen. In dem Sinn soll er das Leben von John Connor (J.C.!) schützen, der es in der Zukunft als Untergrundkämpfer erfolgreich mit den Mächten der Erde aufnehmen wird. Sehen wir uns Terminator II mit dem Vorwissen einer christlichen Kultur an, dann ist es möglich, den Film als einen ,rezeptiv-religiösen Film ' zu verstehen. Terminator II enthält dann die Züge einer profanen Christusfigur, die ihr Leben opfert, damit die Welt gerettet wird. Übrigens ist ein sozusagen profan-religiöses filmisches Thema in so vielen Filmen durch die ganze Filmgeschichte hindurch aufzuweisen, daß eine Auflistung unmöglich ist. Ich verweise dazu u.a. auf das ,Lexikon Religion im Film' und andere filmwissenschaftliche Veröffentlichungen zu dieser Thematik. Selbstverständlich lassen sich die oben gegebenen Filmbeispiele noch um ein Vielfaches erweitern.

Obwohl die Macher der hier nur beispielhaft ausgewählten Filme durchaus mit der christlichen Kultur vertraut sein werden, ist - u.a. aufgrund ihrer eigenen Äußerungen in Interviews, aber auch aufgrund eingehender Filmanalysen - anzunehmen, daß der jeweilige Film von ihnen nicht als ein ,produktiv-religiöser Film' entwickelt worden ist. Das religiöse Element ist zwar im Film vorhanden, aber nicht manifest, sondern auf einer nicht immer bewußt wahrzunehmenden latenten Ebene, sozusagen ungewollt, was wiederum nicht heißt, das der Filmemacher bei der Konzeption seines Werkes etwas zuließ, das seinen Entscheidungen entzogen war. Vielmehr können wir davon ausgehen, daß innerhalb der westlichen (Film)kulturen die christliche Kultur auch in der nicht-religiösen Kultur latent vorhanden ist. Der kulturelle Einfluß des Christentums ist in unserer Kultur so stark, daß es m.E. ohne weiteres möglich ist, hier von einer a-religiösen bzw. profanen christlichen Kultur zu sprechen. Eine große Anzahl uns vertrauter, auch meist als profan verstandener kunstgeschichtlicher Bilder haben im Grunde genommen eine christlich-ikonologische Basis. Die relative Vertrautheit eines Zuschauers mit diesen bei ihm auf unbewußter Ebene immer vorhandenen Kulturelementen macht es den Filmemachern möglich, eine semi- oder quasi-religiöse Wirkung mit ihren an sich profanen Filmen zu erreichen, vorausgesetzt der Zuschauer ist in der Lage, diese Kulturelemente adäquat zu verstehen. Ein Teil der Zuschauer ist innerhalb eines bestimmten kulturellen Umfelds im Stande, mittels der ,erkennbaren Analogie' das latent religiöse Element im Film für sich manifest werden zu lassen. Er sieht ein Bild, eine Handlung, ein Objekt und nimmt sozusagen das Religiöse darin wahr, wo ein anderer Zuschauer dieses Religiöse im gleichen Bild, in der gleichen Handlung, im gleichen Objekt nicht wahrnimmt und auch nicht wahrnehmen kann, weil ihm die ,erkennbare Analogie' fehlt. Nur wem dieses latent Religiöse im profanen Film, z.B. mit Hilfe eines 
Filmgesprächs bewußt geworden ist, kann dieses Element adäquat (religiös) verstehen. Da aber die vom Filmemacher verwendeten semiund quasi-religiösen Bilder Teil einer profanen Gesammtkultur sind, ist dieses religiöse Verstehen für das Totalverständnis eines Filmes nicht unerläßlich. Auch ohne die religiöse Konnotation kann der ganze Film von einem Zuschauer durchaus adäquat (profan) verstanden werden. Das latent religiöse Element gibt dem Film für den, der es sehen kann, einen Mehrwert.

Es gibt aber auch eine große Anzahl von Filmen, in denen religiöse Themen auf manifester Ebene aufbereitet sind. Ein paar Beispiele sind Tagebuch eines Landpfarrers (Robert Bresson, F 1950), Das siebte Siegel (Ingmar Bergman, S 1956), La Ultima Cena/Das letzte Abendmahl (Thomas Gutièrrez Aléa, Cuba 1978) oder Die Sonne Satans (Michel Pialat, F 1987). Diese Filme beinhalten eine in der westlichchristlichen Kultur eindeutig als religiös/christlich auszuweisende Geschichte und zeigen bei eingehender Analyse auch ein als christlich zu identifizierendes Konzept. Ob diese aber deswegen schon ,religiöse Filme' genannt werden können, ist für mich gerade die Frage. Denn nur wer mit der entsprechenden christlich-religiösen Kultur vetraut ist, wird mit derartigen Filmen etwas anfangen können. Wer mit dieser Kultur nicht vertraut ist, die grundlegende (biblische) Geschichte (Das letzte Abendmahl) nicht kennt oder nichts über das Wesen des Priesterseins (Tagebuch eines Landpfarrers, Die Sonne Satans) informiert ist, wird diese Filme nur schwer verstehen können.

Es gibt aber noch eine weitere Möglichkeit, nämlich die, in der ein Film mit Hilfe einer profanen Geschichte einen tieferen religiösen Inhalt vermitteln will. Dies sehen wir z.B. in den meisten Filmen des Schweden Ingmar Bergman (z.B. Wilde Erdbeeren (1957), Das Schweigen (1963), Schreie und Flüstern (1972), Von Angesicht zu Angesicht (1972) usw.). Auch Filme von Andrej Tarkowski, Krzysztof Kieslowski, Carlos Saura, Luis Buñuel, Victor Enrice u.v.a., zeigen manifest religiöse Themen auf. $\mathrm{Ob}$ aber diese Filme auch vom Zuschauer als solche - d.h. als religiöse Filme - erkannt werden, hängt wiederum vom Zuschauer $\mathrm{ab}$ - und nicht zu sehr vom Filmemacher. Ein Film, den ein religiöser Mensch religiös nennt, kann für einen anderen Menschen, der dieses religiöse Selbstverständnis nicht hat, profan wirken. Viele indische Filme z.B. wirken auf westliche Zuschauer eher wie melodramatische Schnulzen, wo sie nachweisbar einen tiefen hinduistisch geprägten religiösen Inhalt haben, den wir nicht erkennen können, weil uns der hinduistische Referenzrahmen, d.h. die ,erkennbare Analogie' zu den Filmbildern fehlt.

Schließlich gibt es Filme, die uns Geschichten, die wir kennen, aus einem nachweisbar religiösen Kontext erzählen bzw. die uns Geschichten erzählen, die in einen solchen Kontext aufgenommen werden können. Beispiele dafür sind bekannte italienische oder HollywoodFilme der 50er Jahre wie Der Mantel, Barabbas, Samson and Delilah, Ben 
Hur usw.; Filme, die entweder eine Phantasiegeschichte um eine biblische Figur erzählen und sich so das Prädikat,religiös' zu erwerben erhoffen, oder aber Filme, in denen etwa eine Reliquie (der von den Soldaten verloste Mantel Jesu) oder aber z.B. Jesus selber in einer zentralen Nebenrolle (Ben Hur) eine wichtige Rolle spielt. Hier haben wir es eindeutig mit profanen Filmen um ein religiös vermarktetes Thema zu tun. So ist Der Mantel nichts anderes als eine romantische Liebesgeschichte, situiert in der Zeit nach dem Tode Jesu, aber innerhalb der ersten Christengemeinden im römischen Reich. Zwar enthält der Film eindeutige Verweise auf die christliche Botschaft, aber eine wesentliche Rolle spielt diese im Film nicht. $\mathrm{Ob}$ diese Art von Filmen daher als ,religiös' verstanden werden kann, hängt wiederum vom Zuschauer $\mathrm{ab}$. Daß sie religiös vermarktet werden, wird deutlich, wenn man bedenkt, daß der niederländische konservativ-christliche Fernsehsender Evangelische Omroep (EO) diese Art von Filmen in der Vergangenheit regelmäßig ausstrahlte. ,Christliches' (?) Thema und ,christliches' (?) Konzept schließen allem Anschein nach bei der christlichen Vorstellungswelt der Zuschauer dieser Rundfunkanstalt an.

Zusammenfassend können wir feststellen, daß es vier grundsätzlich verschiedene Arten von Filmen gibt. Dabei gehe ich davon aus, daß zwischen der Thematik und dem Konzept eines Filmes unterschieden werden kann. Die Thematik eines Filmes ist manifest, d.h. mehr oder weniger direkt wahrnehmbar. Es ist die erzählte Geschichte: sichtbar, hörbar, bewußt konstruiert. Es ist sozusagen das "Fahrzeug", mit dem das Konzept transportiert wird. Das Konzept indes umfaßt den latenten Inhalt, die verborgene Botschaft des Filmemachers, das, was er dem Zuschauer ,eigentlich' erzählen will. Es ist im Film auf einer latenten Ebene anwesend und umfaßt den Gedanken, die Idee, die der Regisseur vermitteln will. Sie herauszuarbeiten, ist eine der zentralen Aufgaben der Filmwissenschaft. Dabei geht es um die tiefere Bedeutung, die man dem Film beimessen kann und die auch dem Regisseur oft nur teilweise bewußt ist, nämlich um den Inhalt, der z.T. auf einer unbewußten Ebene aus der Interaktion der an der Produktion Beteiligten entsteht bzw. um das, was der Regisseur sagen will und für das er das ,Fahrzeug' seines Kunstwerkes benutzt. Andererseits kann das Konzept des latenten Inhalts aber auch vom Filmemacher gewollt im Kopf der Zuschauers entstehen, weil er das, was im Film reflektiert wird, bei sich wiedererkennt. Bekannt ist im diesem Sinne die Art und Weise, wie Krzysztof Kieslowski sich meistens weigert, die von ihm gemeinte Bedeutung seiner filmischen Symbole zu offenbaren. Er meint zurecht, $\mathrm{da}$ es der Zuschauer ist, der diesen eine Bedeutung geben soll - und nicht der Regisseur. 
Bringen wir das bisher Gesagte in ein Schema unter, so entsteht folgendes Bild:

religiöse Thematik

religiöses Konzept

profanes Konzept religiöser Film

z.B.Tagebuch eines

Land pfarrers,

Warum Bhodydharma in den Orient

ging,

Das letzte Abend-

mahl

usw.

semi-profaner Film

z.B. Ben Hur, Der

Mantel,

Pontius Pilatus,

die meisten Bibelfil-

me (?) profane Thematik

quasi-profaner Film

z.B. Filme von Bergman, Saura, Buñuel Kieslowski,

Tarkowski

usw. profaner Film

die gängigen

Hollywoodproduktionen

usw.

Hinzu kommen noch eine Anzahl sog. Grenzfälle: Filme, deren Thematik zwar eindeutig und klar als religiös eingestuft werden kann, wobei man aber die Frage stellen sollte, was der Filmemacher mit dem Film beabsichtigt. Diese Frage nach dem Konzept des Filmemachers bringt im Allgemeinen das Problem der klaren Zuordnung mit sich. Die Frage stellt sich z.B. deutlich bei der neuen Bibelfilm-Serie, auf die ich am Anfang dieses Artikels eingegangen bin. Eine erste und vorläufige Analyse der ersten Folgen der Serie (Abraham und Jakob) gaben mir keinen Aufschluß über das Konzept der Filmemacher, und es ist zu befürchten, $\mathrm{da} ß$ auch die anderen Folgen in diesem Punkt nicht deutlicher sein werden. In dieser Hinsicht unterscheidet sich diese Serie nicht von früheren Bibelfilmen; und das ist nicht nur schade, sondern auch eine verpaßte Chance. Denn gerade in dieser Zeit hätte eine derartige Serie auf der Basis eines deutlichen Konzeptes einen ausgezeichneten Beitrag zu der außerkirchlichen Reaktivierung des christlichen Erbgutes in der westlichen Gesellschaft leisten können. Es hätte ein erster Schritt auf dem Electronic Super Highway sein können. Jetzt ist es ein einfaches ,Remake' gescheiterter vergleichbarer Versuche.

Die meisten Bibelfilme sind in dieser Hinsicht typische Beispiele für Filme, die von der Thematik als religiös einzustufen, aber vom Konzept her meist sehr undeutliche, eher profane Produktionen sind. 
Die Frage nach dem Konzept läßt sich meist nur schwer beantworten; es sei denn, der Filmemacher hat sich dazu deutlich geäußert, wie dies beim Genesisprojekt Ende der 70er Jahre geschehen ist. Dieses Bibelfilmprojekt hatte als Ziel, die Bibel von vorne bis hinten zu verfilmen, und zwar in einer solchen Art und Weise, daß das Produkt (eine audiovisuelle Bibel für die, die das Lesen nicht beherrschen) als Evangelisationsmittel hätte benutzt werden können.

Die Frage nach dem Konzept eines Bibelfilmes ist eine durchaus relevante Frage: Will der Filmemacher nur eine bekannte - zufälligerweise biblische - Geschichte neu erzählen? Oder will er mehr als nur das? Wie sich aus einem Forschungprojekt ergibt, haben viele der bekannten und weniger bekannten Jesusfilme überhaupt kein nachweisbares religiöses Konzept. Meist sind es rein profane Filme über eine historisch wichtige religiöse Figur; d.h., daß die Thematik als religiös verstanden werden kann. Wer einen solchen Film in der richtigen religiösen Fassung sieht, kann dabei durchaus zu einem religiösem Verständnis des jeweiligen Filmes kommen. Diese Filme können unter Umständen als religiöse Filme gesehen und verstanden werden, ohne daß sie das aufgrund der obigen Vierteilung wirklich sind.

Wer Martin Scorseses Die letzte Versuchung als einen religiösen Film sehen will, hat dazu von der Thematik des Films her jede Möglichkeit. Wer den Film als die Verfilmung des gleichnamigen Romans von Nikos Kazantakis sehen will, braucht auch nicht enttäuscht $\mathrm{zu}$ werden. Nur wer den Film als eine Bibelverfilmung $\mathrm{zu}$ sehen wünscht, vergißt, daß er dem Film etwas abgewinnen will, was der Film nachweisbar nicht bieten kann (Scorsese beschreibt dies eindeutig in den Anfangsbildern des Filmes). Ähnliches gilt für Franco Zeffirelli's Jesus von Nazareth. Auch dieser Film bietet dem Zuschauer jede mögliche interpretative Freiheit. Wer will, kann ihn als ,religiösen' Film sehen; wer das nicht will, der kann einen recht uninteressanten profanen Film sehen, obwohl Zeffirelli selber den Film als eine Evangelienverfilmung verstanden haben will, und der Film als solcher auch vom Vatikan ,beglaubigt ' worden ist. Bei Monty Pythons Das Leben des Brian (GB 1979) ist eindeutig kein religiöses Konzept vorhanden. Der Film basiert zwar auf der biblischen Jesus-Geschichte, parafrasiert ihn aber in einer solchen Art und Weise, daß ein einzigartiges Produkt entstanden ist, das sich nur schwer im obigen Schema einordnen läßt.

Ausgangspunkt meiner Überlegungen zum ,religiösen Film' war und ist, daß es an sich keinen eindeutig, religiösen Film ' gibt. Ganz so stimmt dies natürlich nicht, denn oben habe ich ja versucht, verschiedene Kriterien aufzuzeigen, unter denen man einen Film als ,religiös' einstufen kann. Ein wichtiger Punkt bleibt aber, daß nicht der Filmemacher oder etwa der Filmkritiker darüber entscheidet, ob ein Film als religiös angesehen werden kann. Es ist der Zuschauer, der diese Entscheidung trifft. Nur dieser ist imstande, die ihm angebotenen Bilder im Rahmen seines eigenen religiösen Verständnisses als religiöse Bilder 
zu verstehen; d.h. daß auch Filme mit Jesus in der Hauptrolle' nicht ohne weiteres als religiöse Filme angesprochen werden können. Genau hier sollte die Diskussion innerhalb der Theologie anfangen. Hier nämlich stellt sich die Frage nach den Kriterien, nach denen ein Film, der eine biblische, religiöse oder aber eine profane Geschichte erzählt, als religiöser Film einzustufen ist. Die Frage ist nicht mit einem Verweis auf die typisch religiöse Zugehörigkeit der Hauptfiguren zu beantworten. Wäre es so einfach, so wäre z.B. schon ein einfacher Krimi, in dem ein Priester ermordet wird oder einen Kriminalisten spielt (Father Brown-Serie), allein schon deshalb ein religiöser Film. Dann müßten quantitative Kriterien gefunden werden, nach denen festgestellt werden kann, wie lange eine Szene z.B. mit einem Priester oder einer Nonne dauern müßte, um einem Film das Prädikat,religiös‘ zu verleihen. Dies ist eine hoffnungslose und praktisch sinnlose Angelegenheit.

Wer die Frage nach dem religiösen Film stellt, stellt sich eine qualitative, inhaltsanalytische Frage. Nur die genaue analytische Interpretation von einer großen Anzahl von Filmen kann die Frage beantworten, ob und unter welchen Kriterien es einen religiösen Film gibt. 100 Jahre Filmgeschichte lassen es an der Zeit erscheinen, daß sich Theologen und Filmwissenschaftler zusammentun, um das Konzept ,religiöser Film' auf diesem Hintergrund neu zu überarbeiten und die Kriterien aufzubereiten, die diesem in sich wichtigen und interessanten filmischen Genre Bedeutung verleihen. Bis es aber soweit ist, d.h. bis die Kriterien eingehend entwickelt und diskutiert worden sind, gibt es für mich keinen religiösen Film.

\section{SUMMARY: The (religious) film? He doesn't still exist!}

The author argues that religious film does not exist unless the criteria to call a film, religious are well discussed and developed. He argues that up to then every film can be called religious as long as it refers to a reality, that is called ,religious' by the filmviewer. Not the filmmaker but the filmviewer decides on wether a film should be called religious or profane. The only criteria that exist today are the subjective criteria of the viewers. Therefore, this article deals with the relationship between the film and its recipients, that cannot be discussed only by ,objective criteria'. Nevertheless, ongoing research by both theologians and filmresearchers should develop more so called ,bordercriteria' for the genre of the religious film. Up to then there isn't such a genre as a ,religious film'.

\section{RÉSUMÉ: Le film (religieux)? Il n'existe absolument pas!}

L'auteur fait observer que chaque film pourrait être dit "religieux" quand il se rapporte à une réalité que le spectateur considère comme "religieuse". C'est la raison pour laquelle le producteur ne fait pas la différence si un film est un film "religieux". C'est le spectateur qui fait cette différence. Aujourd hui, un film ne peut être jugé que d'après les critères subjectifs des spectateurs. C'est pourquoi, dans cet article, il est question de la relation complexe entre film et 
spectateur, relation que l'on ne peut pas seulement décrire avec des „critères objectifs". A l'avenir, les théologues et les producteurs de films devront quand même essayer de trouver ensemble des criterres objectifs, c'est à dire des critères appelés "critères limites" pour le genre du film religieux. Jusqu'à présent, ce genre n'existe pas encore.

RESUMEN: ¿Películas religiosas? Películas de ese tipo no existen!

El autor señala que toda película puede ser denominada „religiosa“, si ésta se refiere a una realidad percibida como "religiosa" por el espectador/ receptor. De ahí que no sea el productor el que diferencia si una película es religiosa o no, sino el espectador. Hoy en día, una película sólo puede ser juzgada según los „criterios subjetivos" de los espectadores. Por eso este artículo trata ante todo la compleja relación entre una película y el receptor, cuestión que no se deja explicar solamente con "criterios objetivos". A pesar de eso, en el futuro deberían buscar juntos teólogos y productores criterios objetivos, es decir, buscar los llamados "critios límites" para el género de las películas religiosas. Hasta hoy tal género no existe. 